\title{
Radiation Hydrodynamics in Pulsating Stars
}

\author{
Michael U. Feuchtinger \& E. A. Dorfi \\ Institut für Astronomie der Universität Wien \\ Türkenschanzstraße 17, A-1180 Wien, Austria
}

\begin{abstract}
We present nonlinear radiation hydrodynamical calculations of Cepheid envelopes carried out using the method described in Dorfi \& Feuchtinger (1991, A\&A 249,414). The radiative transport is treated within the grey Eddington approximation and all equations are discretised in conservative form on an adaptive mesh which is solved simultaneously with the physical equations. The resulting system of nonlinear algebraic equations is solved implicitly to avoid the restrictive Courant-Friedrichs-Lewy time step condition. In order to treat schock waves we use the artificial tensor viscosity developed by Tscharnuter \& Winkler $(1979$, Comp.Phys.Comm. 18, 171) which is suitable for spherically symmetric problems. For the opacity we employ the latest OPAL tables (Rogers and Iglesias, 1992, ApJ, submitted); the equation of state corresponds to the standard evolution calculations according to Baker \& Kippenhahn (1962, Zeitschr. f. Astrophys. 54,114).

We start from an initial hydrostatic model whose parameters correspond to a typical Cepheid $\left(5 M_{\odot}, 3000 L_{\odot}, 5600 \mathrm{~K}\right)$. The subsequent dynamical evolution is initiated by the esccitation of oscillations at the ionization zones in the stellar atmosphere where the opacity changes by several orders of magnitude. After an initial relaxation caused by discretisation errors and numerical noise, a periodic pulsation develops. Each pulsation is accompanied by a shock wave which propagates through the stellar atmosphere. A typical pulsation cycle is covered by about 70 time steps and the obtained periods lie in the range of 1 to 40 days.

We discuss in detail the excitation of the oscillations and the structure of the model during a pulsation cycle.
\end{abstract}

This work is supported by the Österreichischer Fonds zur Förderung der wissenschaftlichen Forschung under project number P8758. 